Preaxial Polydactyly in Sost/Sostdc1 Double Knockouts

C. M. Yee, N. M. Collette, G. G. Loots

August 1, 2011 
This document was prepared as an account of work sponsored by an agency of the United States government. Neither the United States government nor Lawrence Livermore National Security, LLC, nor any of their employees makes any warranty, expressed or implied, or assumes any legal liability or responsibility for the accuracy, completeness, or usefulness of any information, apparatus, product, or process disclosed, or represents that its use would not infringe privately owned rights. Reference herein to any specific commercial product, process, or service by trade name, trademark, manufacturer, or otherwise does not necessarily constitute or imply its endorsement, recommendation, or favoring by the United States government or Lawrence Livermore National Security, LLC. The views and opinions of authors expressed herein do not necessarily state or reflect those of the United States government or Lawrence Livermore National Security, LLC, and shall not be used for advertising or product endorsement purposes.

This work performed under the auspices of the U.S. Department of Energy by Lawrence Livermore National Laboratory under Contract DE-AC52-07NA27344. 


\title{
Preaxial polydactyly in Sost/Sostde1 double knockouts
}

\author{
Cristal Yee \\ University of California, Merced \\ Gabriela G. Loots \\ Ph.D., Molecular and Cell Biology \\ Biomedical Scientists at LLNL
}

Lawrence Livermore National Laboratory, Livermore, CA

July 29, 2011 


\begin{abstract}
In the United States, $\sim 5 \%$ are born with congenital birth defects due to abnormal function of cellular processes and interactions. Sclerosteosis, a rare autosomal recessive disease, causes hyperostosis of the axial and appendicular skeleton, and patients present radial deviation, digit syndactyly, nail dysplasia, and overall high bone mineral density. Sclerosteosis is due to a loss of function of sclerostin (Sost). Sost is a Wnt (abbrev.) antagonist; when mutated, nonfunctional Sost results in hyperactive osteoblast activity which leads to abnormal high bone mass. Previous studies have shown that Sost overexpression in transgenic mice causes reduced bone mineral density and a variety of limb phenotypes ranging from lost, fused, and split phalanges. Consistent with clinical manifestations of Sclerosteosis, Sost knockout mice exhibit increased generalized bone mineral density and syndactyly of the digits. Sostdc1 is a paralog of Sost that has also been described as an antagonist of Wnt signaling, in developing tooth buds. Unlike Sost knockouts, Sostdc1 null mice do not display any limb abnormalities. To determine if Sost and Sostdc1 have redundant functions during limb patterning, we examined $\mathrm{Sost}^{-/}$; Sostdc $1^{-/-}$mice determined that they exhibit a novel preaxial polydactyly phenotype with a low penetrance. LacZ staining, skeletal preparations, and in situ hybridization experiments were used to help characterize this novel phenotype and understand how this phenotype develops. We find Sost and Sostdc1 to have complementary expression patterns during limb development, and the loss of their expression alters the transcription of several key limb regulators, such as Fgf8, Shh and Grem.
\end{abstract}

\title{
Introduction
}

Sclerosteosis is a rare autosomal recessive disease that causes hyperostosis to the axial skeleton and appendicular skeleton. Patients with Sclerosteosis appear to have radial deviation, hand malformations (commonly syndactyly of digit 2 and 3), nail dysplasia, overall high bone density, and secondary neurological defects (Fig1A, B, C). Some of these secondary neurological defects, such as hearing loss and constriction of the optic nerve, can escalate to sudden death caused by increased intracranial pressure. This rare hyperostosis disease is due to loss of sclerostin (SOST), which causes hyperactive osteoblast activity.

Sost has been initially described as a BMP (Bone Morphogenic Protein) antagonist, but in the recentyears Sost has emerged as a Wnt antagonist (Collette et al., 2010). Altering the function of Sost results in increased or decreased Wnt signaling. For example, using Sost transgenic mice causes osteopenia and reduced bone mineral density (BMD) in both the appendicular and axial skeleton (Loots et al., 2005) due to reduced Wnt signaling in bone. Furthermore, these transgenic mice show a variety of phenotypes ranging from fused, split, to missing phalanges due to dosage-dependent overexpression of Sost (Collette et al., 2010). Alternatively, knocking out Sost results in a growth increase of bone formation and digit syndactyly phenotype (Fig1).

Sostdc1 (Sost domain-containing protein 1; aka Sostl, USAG-1, Wise, ectodin) is a paralog of Sost and an antagonist of the LRP receptor in tooth buds (Kasai et al., 2005). Sost and Sostdc 1 share 40\% identity at the DNA sequence level and a 55\% protein similarity and initial studies have reported Sostdcl to influence teeth. In one instance, 
Sostdc1 knockout mice generated excessive numbers of incisors as well as affected Fgf and Shh signaling in the developing tooth bud (Kassai et al., 2005) (Fig2). Since Sostdc1 is paralogous to Sost and affects the Fgf and Shh signaling when altered, these two genes may have similar functional roles during limb development. We have examined $\mathrm{Sost}^{-/}$; Sostdc $1^{-/}$double knockout mice and found them to exhibita variably penetrant preaxial polydactyly phenotype, detected as early as E12.5 (Fig3). This may suggest that Sostdc1 and Sost plays similar, redundantroles, interfering with the same signaling pathway in the limb, which leads to polydactyly.

This preaxial polydactyly phenotype in the Sost ${ }^{-/}$; Sostdc $1^{-/-}$double knockout mice has led to questions such as: (1) where are Sost and Sostdc1 expressed? (2) are Sost and Sostdc1 interfering with the same signaling pathway? (3) how is digit 1 determined during limb development and digit formation? To further our investigation, we have designed three sets of experiments that would uncover Sostdc1 role and contribution in limb deformity. The first aim is to locate where Sost and Sost dc 1 are expressed under normal developmental conditions, via LacZ staining of organs, bone segments, and forelimb buds. Locating where these two genes are will assist in determining of where and when these two genes are expressed. Second of all, we aim to compare and analyze cartilage condensation and digit formation throughout developmental time points of wildtype, Sost knockout, Sostdc1 knockout, and Sost/Sostcd1 double knockout mice via skeletal preparations. Comparing skeletal preparations presents limb development and outlines when the polydactyly phenotype begins developmentally. Finally, the third aim is to analyze expression patterns along the anterior posterior regions via in situ experiments of different markers to determine what signals are responsible for digit 1 (thumb) formation and how other genes are effected by Sost and Sostdc1 alterations.

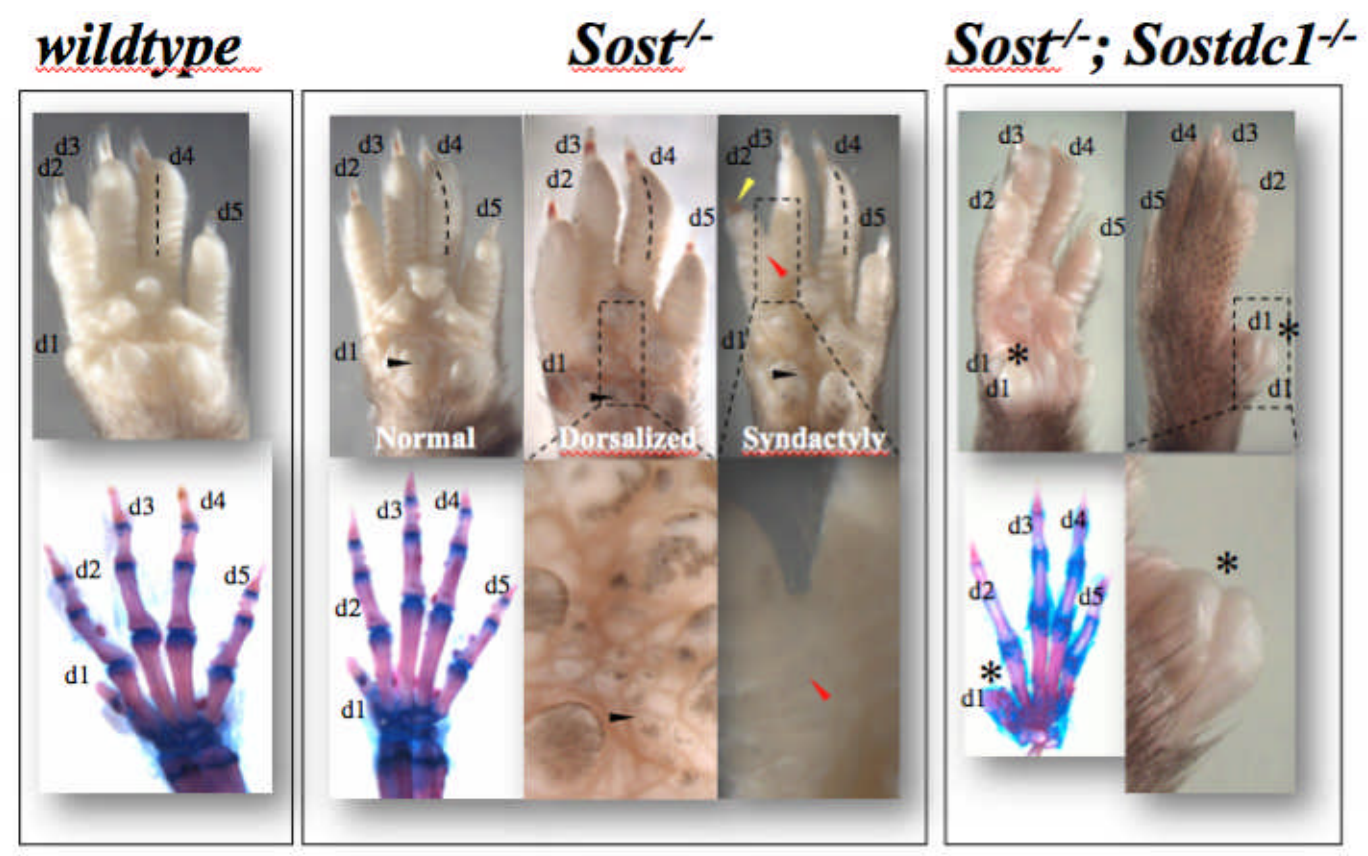

Figure 1. Phenotypes seen in Sost ${ }^{-/}$and $\operatorname{Sost}^{-/}$; Sostdc1 $1^{-/}$double mutant mice. Wildtype, some Sost ${ }^{-/}$, and Sostdc $1^{-/}$mice (not shown), appear to have normal autopods. Some Sost ${ }^{-/}$mice develop autopod defects similar to those seen in sclerostosis patients, consisting of dorsalization of the ventral autopod (black arrowheads and inset) soft tissue syndactyly (red arrowheads and inset), nail dysplasia (yellow arrowhead), 
and radial deviation (curved lines). Sost ${ }^{-/}$; Sostdc $1^{-/}$double mutants show preaxial polydactyly, or duplication of anterior digits (asterisks and inset). Skeletal preparations of adult autopods are also shown.

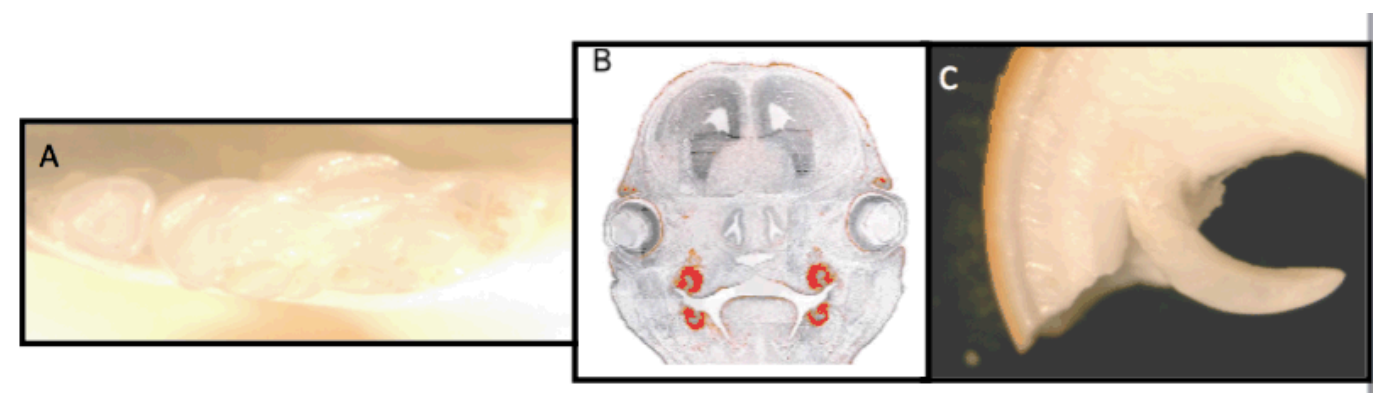

Figure 2. Sostdc1 is an antagonist of the LRP receptors in developing tooth bud. (A) Sostdc $1^{-/}$mice develop a fused molar phenotype. (B) A cross-section of an embryo's cranium shows Sostdc1 being expressed in the tooth buds. (C) Sostdc $1^{-/}$mice alters the Wnt and Shh signaling, causing supernumerary incisors.
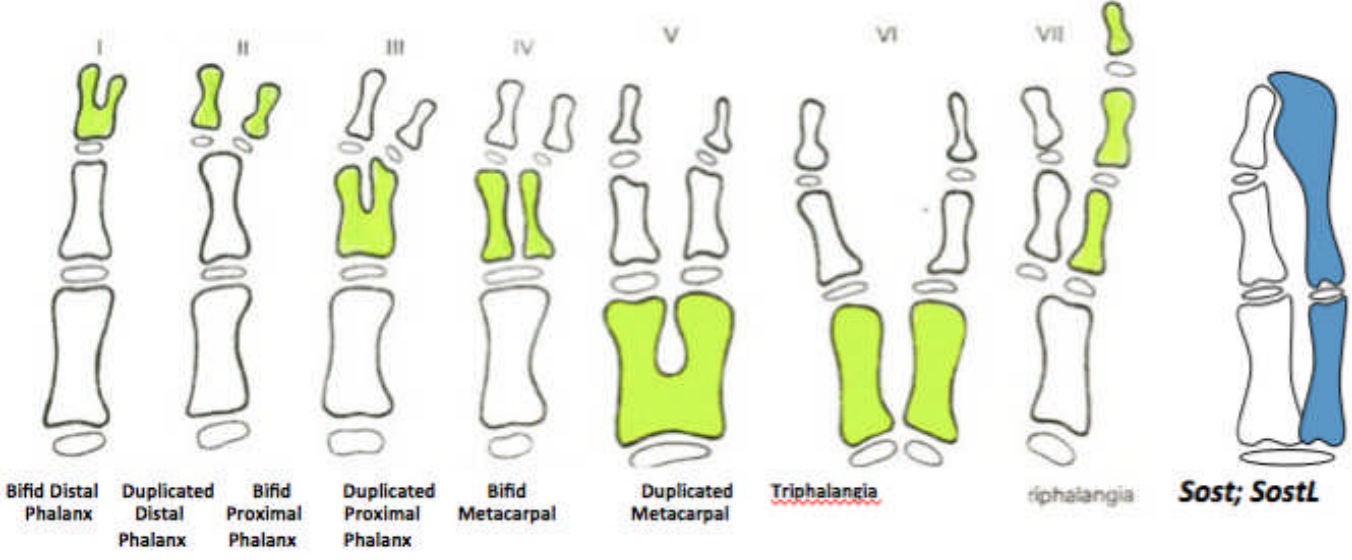

Figure 3. The polydactyly phenotype has been categorized under the standard VII classification. To the right, displays the novel preaxial polydactyly expressed in $\mathrm{Sost}^{-/} ;$Sostdc $^{-/-}$mice.

\section{Results and Discussion}

\section{Sost and Sostdc1 Expression}

Our first goal was to characterize Sost and Sostdc1 expression pattern during embryonic development. The Sost ${ }^{-/}$and Sostdc $1^{-/-}$strains available in our laboratory have been generated through a knock-in strategy, where the LacZ gene has replaced the endogenous transcript, and is transcribed in frame from the endogenous promoter, such that LacZ expression faithfully recapitulates the expression of the knocked out gene. Performing LacZ staining on a cross-section of $\mathrm{Sost}^{-/}$and Sostdc ${ }^{-/-}$forelimb reveals adjacent, non-overlapping expression of Sost and Sostdc1 (Fig4). 


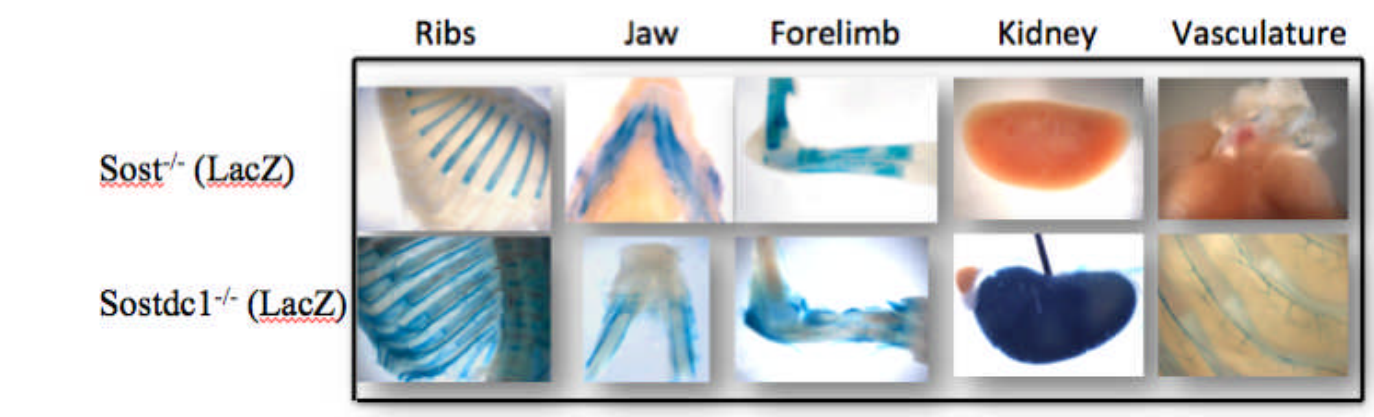

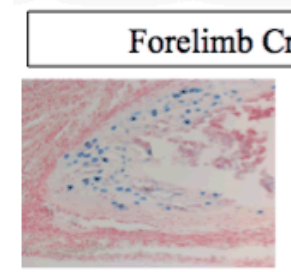

Sost $^{-/}(\mathrm{LacZ})$

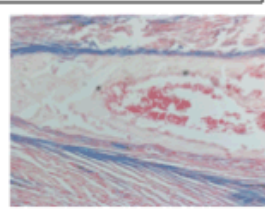

Sostdc1 ${ }^{-}(\operatorname{LacZ})$
Figure 4. LacZ staining on Sost ${ }^{-/}$and $\mathrm{Sostdc}^{-/ /}$mice displays adjacent, non-overlapping expression patterns throughout various parts of the mouse (A). Taking a crosssection of the forelimb shows Sost is expressed in osteocytes while Sostdc1 is located within the other tissues

The adjacent, non-overlapping expression of Sost and Sostdc1 is not only seen in the forelimbs of mice, but as well as in the ribs, jaw, kidney, and vasculature. Comparing Sost and Sostdc1 expression discloses Sost primarily sited within the bone, whereas Sostdc1 is exposed in the surrounding tissue of the bone. Since we are largely interested in the preaxial polydactyly phenotype of Sost ${ }^{-/ ;} \operatorname{Sostdc}^{-/-}$mice, we concentrated on the forelimbs of the mice. Using the LacZ staining method, we performed LacZ stain on whole embryos between E9.5 to E13.5 (Fig5). Sost expression appears in the developing limb starting at E9.5 as opposed to Sostdc1, which begins to emerge at E11.5. Cross sections were made of the forelimb buds to reveal that Sost is detected in the ectoderm and digit tips of the developing limb and Sostdc1 is distinguished in the limb mesenchyme. Thus, these genes in combination are being expressed in co mplementary domains of the limb during limb patterning. 
Figure 5. LacZ stain comparing Sost and Sostdcl endogenous expression in the developing embryo. Genes were knocked out and replaced by a $L a c Z$ cassette to track endogenous gene expression via tissue LacZ stain. Expression in E9.5 to E13.5 whole embryos, Sost expression in top row (A'E'), Sostdcl expression in bottom row. Overall, expression patterns are adjacent and relatively non-overlapping. (F) LacZ stain comparing endogenous expression in the developing limb. Whole limb dorsal view, and cut sections show Sost expression confined to the ectoderm (left panels) while Sostdcl has significant expression in the mesenchyme (right panels), positioning the combined expression domains of these genes to affect limb patterning.

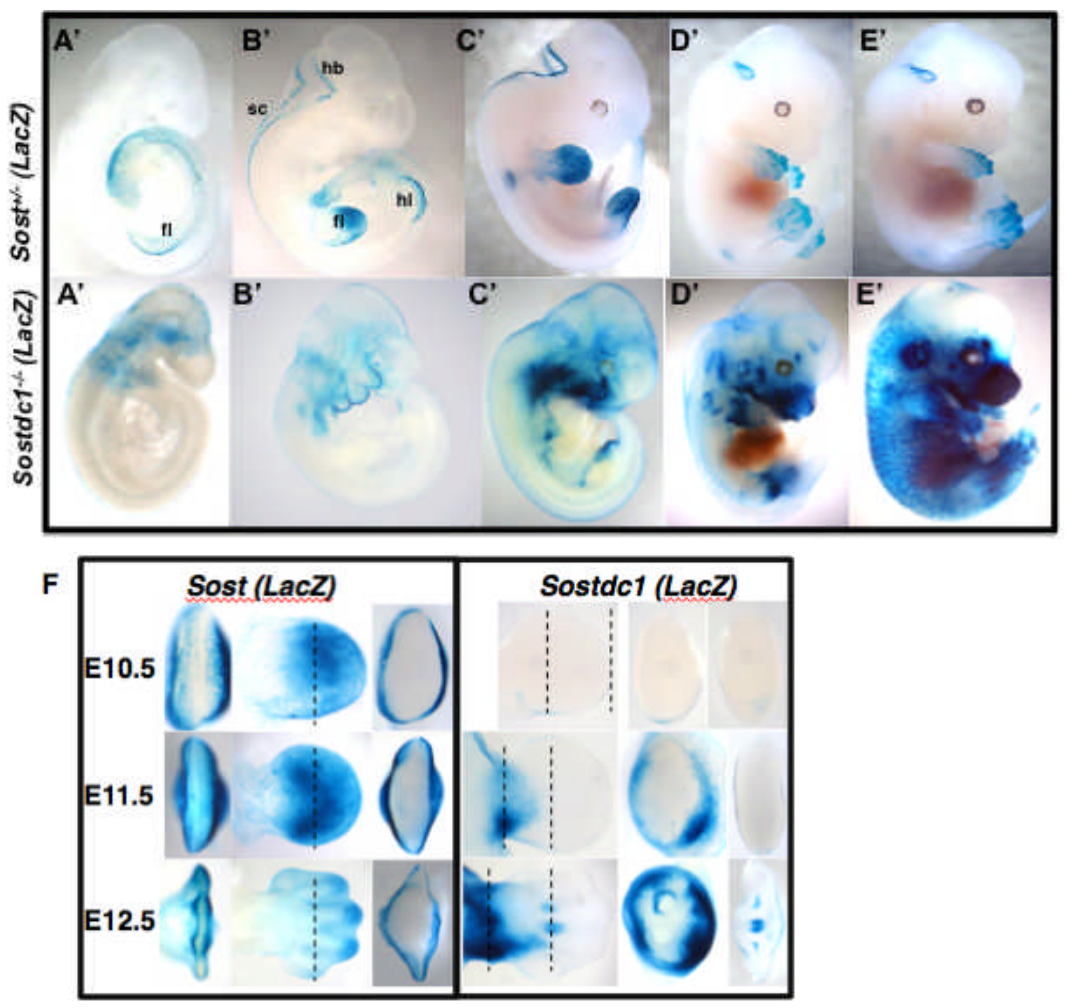

\section{Preaxial Polydactyly Phenotype}

Once the pattern of both Sost and Sostdc1 was determined, skeletal preparations were used to visualize when this preaxial polydactyly phenotype occurs. With the understanding that cartilage forms before bone and acting as a skeletal frame for bone development, analyzing cartilage development prior to the double thumb phenotype will give more insight as to how this unusual development emerges. Skeletal preparations with Alcian Blue cartilage staining were prepared on E14.5 embryos (Fig6). Based on the skeletal preparations outlining cartilage patterning defects that lead to the preaxial polydactyly phenotype of Sost ${ }^{-/}$; Sostdc $1^{-/-}$mice, it is clear that shifting Sost and Sostdc1 together triggers a butterfly affect on the underlying mechanism behind normal limb development. Altering the Sost gene or Sostdc1 gene individually does not result in any visible limb phenotype. This could imply that when one gene is nonfunctional, the other ortholog gene compensates for the other. By removing both genes at once, these two nonfunctional genes create a compound affect in a form of double thumbs. 


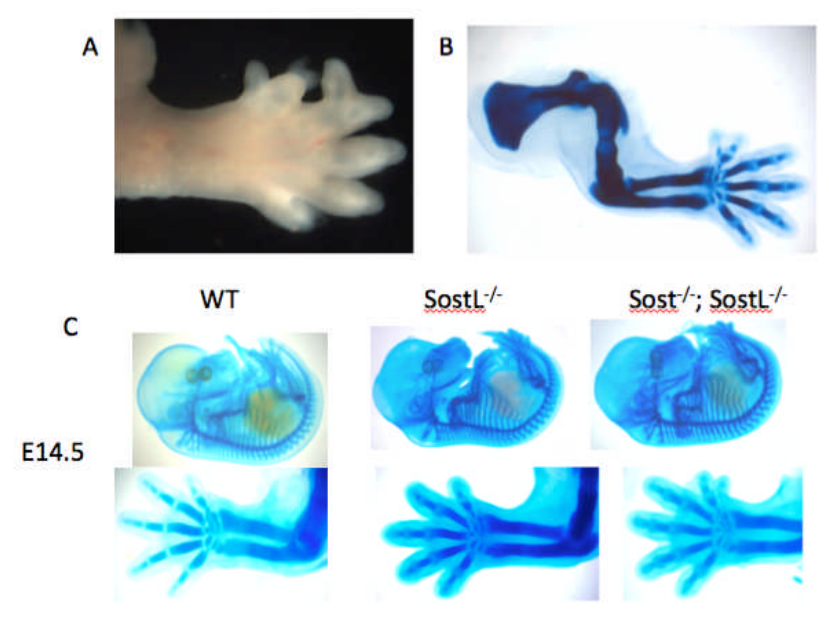

Figure 6. (A) $\operatorname{Sost}_{t^{-/}}$; Sostdc1 $1^{-/}$double mutant mice have variably penetrant preaxial polydactyly, seen embryonically after limb patterning and (B) demonstrate varying degrees of soft tissue and/or skeletal element duplication as seen in the Alcian Blue cartilage stain at E14.5. Embryos were fixed in Bouin's fixative, washed thoroughly in $70 \%$ ethanol:1\% Ammonium hydroxide, and stained overnight in $0.5 \%$ Alcian Blue: 5\% Acetic acid before rinsing and clearing in 1:1 Benzyl Alcohol: Benzyl Benzoate for photography.

\section{Molecular Processes Underlying Limb Development}

Previous research on Sost has shown that it in the limb, it primarily functions to inhibitthe Wnt signaling and alters the expression of Shh, Fgf8, and Greml (Collette et al., 2010). Since limb development involves a complex communication between many genes, in situ methods were used to discover what normal gene expression during limb development are being effected by Sost and Sostdc1 adjustments. We hope to determine genes that Sost and Sostdc1 modulate during limb development as well as what other genes are affected. Antisense Dig-labeled probes and colorimetric detection with BM purple on E10.5 whole mount mouse embryos were used for in situ hybridization experimentations (Fig7). In situ experiments on Sost ${ }^{-/-}$displays an increase in Shh and Fgf8 expression pattern on the posterior and AER region, respectively. In comparison, Sostdc $^{-1-}$ also resulted in an expansion of Shh expression, but restricted Greml significantly. Outcomes from our in situ experiments convey that Sost and Sostdc1does modify expression of genes that regulate the anterior-posterior patterning of the limb. It is important to note that based on the LacZ stains, Sostdc1 appears at a later time point (E11.5) in contrast to Sost. Thus, in situ hybridizations must be performed at E11.5 to obtain a supplementary effect upon genes in the double knockouts based on Sostdc1 contribution. Additionally, E10.5 forelimb buds do not show anterior limb markers to be altered by Sost nor Sostdc1 knockouts, but most likely illustrations the onset of limb patterning. We expect to see a difference on anterior markers due to Sost and Sostcd1 knockouts at a later time frame between E11.5 and E12.5 where digit specification and separation begins. 


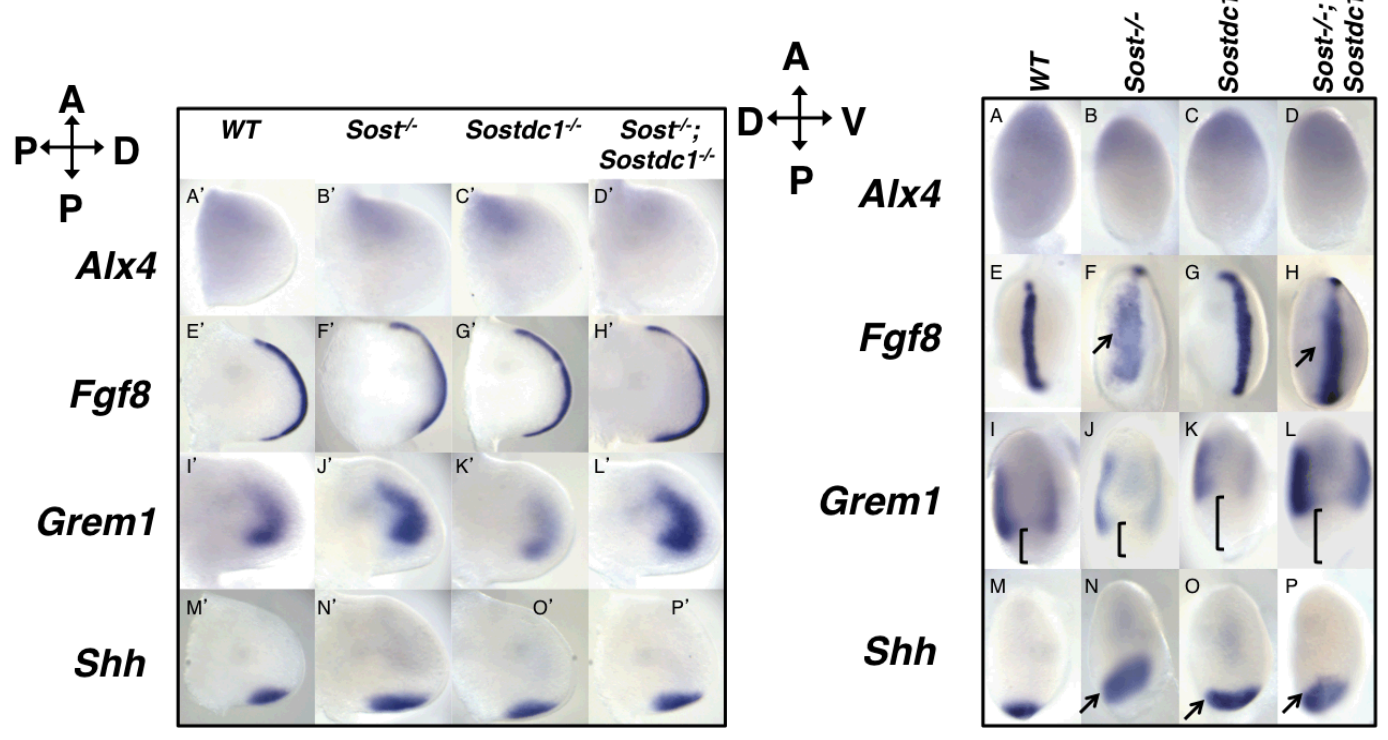

Figure 7. Whole mount in situ hybridization of limb bud markers using antisense Diglabeled probes and BM purple on E10.5 mouse embryos. (A-P) Apical ectodermal ridge (AER) views. (A-D) Alx4 expression shows no disruption of the anterior limb field. (E-H) Fgf8 shows dramatic expansion and disorganization in $\mathrm{Sost}^{-/}$, and to a lesser extent, in $\mathrm{Sost}^{-/}$; Sostdc1 $1^{-/}$embryos along with loss near the anterior margin of expression (arrows, F and H). (I-L) Grem 1 expression shows greater restriction in Sostdc1 $1^{--}$and Sost $t^{--}$; Sostdc 1 ${ }^{--}$embryos (brackets, K and L). (M-P) Shh expression shows possible expansion in all mutant embryos. (A'-P') Dorsal views of the same limb buds for orientation and comparison.

\section{Conclusion}

In summary, Sost and Sostdc1 are paralogous genes that both function as Wnt antagonists, influencing bone and tooth formation, respectively. Our previous research has unraveled that overexpression of Sost causes osteopenia as well as loss of digit formation, while Sost knockout mice have an increase in bone mineral density. Incorporating Sost knockout with Sosdc1 knockout produces a compound axial polydactyly phenotype with a low penetrance in mice. Uncovering the mechanism behind this compound phenotype is an important step in comprehending limb development and limb defects. Knocking in LacZ gene in Sost knockout and Sostdc1 knockout mice helped to trace gene expression throughout the body. Sost and Sostdc1 have adjacent, non-overlapping expressions evident in a cross section of the forelimb, ribs, jaw, kidney, and vasculature. Whole mount LacZ stains throughout E9.5 to E13.5 exposes this non-overlapping Sost and Sostdc1in the forelimb bud; with Sost being located in the ectoderm and digit tips and Sostdc1 being expressed in the mesoderm. Skeletal preparations depicted the axial polydactyly phenotype due to the loss of function in both Sost and Sostdc1 gene by E14.5; which contributed to our hypothesis that Sost and Sostdc1 are engaged in limb development with distinctive roles that work cohesively for proper development of the forelimb. Our last set of experiments utilizing in situ 
hybridization displays Sost and Sostdc1 individually affecting other genes involved in limb development and provides additional support that $\mathrm{Sost}^{-1}$; $\mathrm{Sosdc}^{-/-}$yields double thumb phenotype through a rippling signal effect. To determine Sostdc1 impact in greater details, our next goal is to look at Sostdc1 influence on gene markers at E11.5 and E12.5 as well as to examine anterior markers.

\section{Methods}

\section{Whole-mount in situ hybridization}

Whole-mount in situ hybridizations was carried out using standard procedures (Khokha et al., 2003). Species-specific sequences of human and mouse Sost were cloned from the 3'UTR region of each cDNA (human Sost NM_02537.2; mouse sost NM_024449.4). Briefly, digoxigenin-labeled antisense RNA probes were generated to the desired RNA sequence and hybridized to whole-mount embryos. Expression was visualized by biding BM Purple (Roche). Antisense RNA probes for Grem l (MluI-SacII fragment of NM_011824), Fgf8 (PstI 3'cDNA and UTR fragment of NM_010205; see Crossley and Martin, 1995), and Shh (MscI-NarI fragment of NM_009170; see Echelard et al., 1993) were generated as described (Hogan et al., 1994) with the following modification: proteinase $\mathrm{K}$ digestion was omitted for ectodermal or AER probes. A minimum of 4 embryos were used per genotype, per experiment. Wild-type controls were obtained from the same litter, whenever possible.

\section{LacZ stains embryos}

Genetically modified mice genes were knocked out and replaced with LacZ to track Sost and Sostdc1 expression via tissue LacZ stain. LacZ stains embryos were dissected into ice-cold 1x phosphate-buffered saline (PBS), $\mathrm{pH}$ 7.3. Embyros were fixed in $2 \%$ paraformaldehyde, $0.2 \%$ glutaraldehyde in $1 \times \mathrm{PBS}, 2 \mathrm{mM} \mathrm{MgCl}_{2}$ at $4{ }^{\circ} \mathrm{C}$ for 30 minutes to 1 hour, followed by extensive rinsing in $1 \mathrm{x} \mathrm{PBS,} 2 \mathrm{mM} \mathrm{MgCl}_{2}$. Embryos were stained for 4 hours $(\mathrm{BatGal})$ at RT or overnight $\left(\mathrm{Sost}^{-/}\right)$at $4^{\circ} \mathrm{C}$ in X-gal stain: $1 \mathrm{mg} / \mathrm{ml} \mathrm{X-}$ gal, $\mathrm{MgCl}_{2}, 5 \mathrm{mM}$ EGTA, $0.02 \%$ Nonidet $\mathrm{P}-40,5 \mathrm{mM}$ potassium ferrocyanide, $5 \mathrm{mM}$ potassium ferricynaide, in 1x PBS, pH 7.3. After staining, embryos were postfixed in $4 \%$ paraformaldehyde in $1 \mathrm{x} \mathrm{PBS}, \mathrm{pH} 7.3$ at $4{ }^{\circ} \mathrm{C}$, and then cleared in glycerol for photography.

\section{Skeletal preparations}

Skeletal preparations were made of adult mouse using Alcian Blue 8GX for cartilage and Alizarin Red S for bone following established protocols. E14.5 mouse embryos used Alcian Blue 8GX for cartilage staining only. Embryos were dissected free of internal organs, and skin, and fixed in $95 \%$ ethanol followed by stain in $0.05 \%$ Alcian Blue 8GX, 80\% ethanol, 20\% glacial acetic acid. Excess stain was washed out, and embryos were then stained in $60 \mathrm{mg} / \mathrm{L}$ Alizarin Red $\mathrm{S}, 1 \%$ potassium hydroxide $(\mathrm{KOH})$, $25 \%$ glycerol followed by clearing in $1 \% \mathrm{KOH}$ and $25 \%$ glycerol and storage in glycerol for photography. For skeletal preparation, whole litters were analyzed. 


\section{Acknowledgements}

We would like to thank the National Institutes of Health (NIH), Knock-Out Mouse Program (KOMP), Drs. David Valenzuela and Aris Economides of Regeneron and Dr. Chris Paszty of Amgen for providing the Sost knockout mice. National Nuclear Security Administration (NNSA) Consortium Internship Program for supporting Cristal Yee. G.G.I., N.M.C., and D.M. were supported by NIH grant HD47853. This work performed under the auspices of the U.S. Department of Energy by Lawrence Livermore National Laboratory under Contract.

\section{References}

Collette, N.M., Genetos, D.C., Murugesh, D., Harland, R.M., Loots, G.G., 2010. Genetic evidence that Sost inhibits WNT signaling in the limb. Developmental 342, 169-179.

Crossley, P.H., Martin, G.R., 1995. The mouse Fgf8 gene encodes a family of polypeptides and is expressed in regions that direct outgrowth and patterning in the developing embryo. Development 121, 439-451.

Hogan, B.L., Beddington, R., Costantini, F., Lacey, E., 1994. Manipulating the Mouse Embryos: A Laboratory Manual ( $2^{\text {nd }}$ Edition). Cold Spring Harbor Press, New York.

Kassai, Y., Munne, P., Hotta, Y., Penttila, E., Kavanagh, K., Ohbayashi, N., Takada, S., Thesleff, I., Jernvall, J., Itoh, N., 2005. Regulation of mammalian tooth cusp patterning by ectodin. Science 309, 2067-2070.

Khokha, M.K., Hsu, D., Brunet, L.J., Dionne, M.S., Harland, R.M., 2003. Greamlin is the BMP antagonist required for maintenance of Shh and Fgf signals during limb patterning. Nat. Genet. 34, 303-307.

Loots, G.G., Kneissel, M., Keller, H., Baptist, M., Chang, J., Collette, N.M., Ovcharenki, D., Plajzer-Frick, I., Rubin, E.M., 2005. Genomic deletion of a long-range bone enhancer misregulates sclerostin in Van Buchem disease. Genome Res. 15, 928-935. 